\title{
Barriers and enablers to influenza vaccination uptake in adults with chronic respiratory conditions: applying the behaviour change wheel to specify multi-levelled tailored intervention content
}

\author{
Allyson J. Gallant ${ }^{a}$, Paul Flowers ${ }^{a}$, Karen Deakin ${ }^{a}$, Nicola Cogan ${ }^{a}$ (D), Susan \\ Rasmussen $^{a}$ (D), David Young ${ }^{b}$ and Lynn Williams ${ }^{a}$
}

${ }^{\text {aS }}$ chool of Psychological Sciences \& Health, University of Strathclyde, Scotland, UK; ${ }^{b}$ Department of Mathematics and Statistics, University of Strathclyde, Scotland, UK

\section{ABSTRACT}

Objective: To specify intervention content to enhance influenza vaccination uptake among adults with chronic respiratory conditions using the Behaviour Change Wheel (BCW).

Design: Cross-sectional, multi-modal data collection and theory-informed analysis and expert stakeholder engagement.

Methods: Content analysis was used to identify barriers and enablers to influenza vaccination from nine focus groups $(n=38)$, individual interviews $(n=21)$ and open-ended survey responses $(n=101)$. The Theoretical Domains Framework (TDF) and the BCW were used to specify evidence-based and theoretically-informed recommendations. Expert stakeholders refined recommendations using the Acceptability, Practicability, Effectiveness, Affordability, Side-effects, and Equity (APEASE) criteria to yield a range of potentially actionable ideas.

Results: TDF analysis identified perceptions of vaccine side effects (beliefs about consequences [BACons]) was the most common barrier to vaccination, followed by time constraints (environmental context and resources [ECR]) and fear of needles (Emotion). Enablers included protection from influenza (BACons), receiving reminders (ECR) and support from others (Social Influences). These factors mapped to seven BCW intervention functions and 22 behaviour change techniques.

Conclusions: Factors affecting vaccine uptake are multifaceted and multileveled. The study suggested a suite of complementary multi-level intervention components to enhance vaccination uptake involving a range of diverse actors, intervention recipients and settings.

Abbreviations: APEASE: Acceptability, Practicability, Effectiveness, Affordability, Side-effects, and Equity; BACons: beliefs about consequences; BCT: behaviour change techniques; BCTT: behaviour change technique taxonomy; ; BCW: behaviour change wheel; COVID-19: Coronavirus Disease; ECR: environmental context and resources; GP: general practitioner; HCP: healthcare provider; TDF: theoretical domains framework

\section{ARTICLE HISTORY}

Received 9 April 2021

Accepted 13 July 2021

\section{KEYWORDS}

Influenza vaccination; vaccine hesitancy; behaviour change wheel; theoretical domains framework; behaviour change techniques

CONTACT Lynn Williams Iynn.williams@strath.ac.uk E School of Psychological Sciences \& Health, University of Strathclyde, 40 George Street, Glasgow, Scotland G1 1QE, UK.

(1) Supplemental data for this article is available online at https://doi.org/10.1080/08870446.2021.1957104. 


\section{Introduction}

Seasonal influenza remains a significant public health threat. The most effective way of preventing seasonal influenza is through vaccination, which is offered to particular at-risk groups (e.g. individuals with chronic illnesses). However, a large number of those eligible to receive the seasonal influenza vaccination decide against vaccination (Jorgensen et al., 2018). The term 'vaccine hesitancy' refers to the 'delay in acceptance or refusal of vaccines despite availability of vaccine services' (MacDonald \& the SAGE Working Group on Vaccine Hesitancy, 2015). Seasonal influenza vaccination uptake, even among those who are at high risk (e.g. those with chronic illnesses), is typically less than $50 \%$ in most European countries, which falls substantially below the World Health Organisation target of 75\% (Jorgensen et al., 2018). In Scotland, where the present study was conducted, seasonal influenza vaccine uptake has declined considerably in recent years in the under 65-year-old clinical risk group (i.e. those who are under the age of 65 but receive the vaccination due to an underlying chronic illness). Specifically, within the chronic respiratory disease group, who form the largest proportion of this under-65 clinical risk group, uptake was 44.6\% in 2018-19 (Health Protection Scotland, 2020). It is therefore necessary for research to examine the barriers and enablers to vaccine acceptance in order to inform strategies to address vaccine hesitancy (Brewer et al., 2017).

The reasons for vaccine hesitancy are complex and involve psychological, social and contextual factors (Brewer et al., 2017). Sociodemographic factors, such as age, education and ethnicity, have been inconsistently linked to uptake (Schmid et al., 2017). Contextual factors, relating to how the vaccination programme is delivered and the resulting practical barriers that individuals face in terms of the convenience of getting vaccinated also play a role. However, there is increasing recognition of the role that psychological processes, such as perceived susceptibility and perceived severity, and social norms, play in shaping vaccination behaviour (Schmid et al., 2017).

In their review, Schmid et al. (2017) identified that psychological theories have rarely been applied in understanding the psychological factors influencing influenza vaccine uptake. In those studies that do use theoretically-derived psychological factors, the vast majority of studies have used the Health Belief Model (Borthwick et al., 2021). Applying theory in the design of multi-component interventions has been shown to improve the intervention effectiveness (Craig et al., 2008). To simplify the use of behaviour change theories in designing behaviour change interventions, various behaviour change tools, including the Theoretical Domains Framework (TDF), the Behaviour Change Wheel (BCW) and the Behaviour Change Technique Taxonomy (BCTT) have been developed. The TDF is a behavioural framework of 14 domains and considers the individual psychological, social and environmental influences on behaviours (Atkins et al., 2017; Cane et al., 2012; Lawton et al., 2015). The BCW is comprised of three layers to provide a comprehensive and systematic approach to designing behaviour change interventions (Michie et al., 2014). Ninety-three individual BCTs (e.g. instruction on how to perform the behaviour) grouped into 16 categories (e.g. shaping knowledge) comprise the BCTT to identify the 'observable and replicable' components of interventions (Michie et al., 2013). Applying behaviour change tools in the design and implementation of complex interventions can assist with understanding an interventions mechanisms of change (Craig et al., 2008; Michie et al., 2014). 
Only two studies to-date have applied the TDF to vaccination behaviour. Recently, Kenny et al. (2020) found that the domains of 'Goals', 'Intentions', 'Social influences', and 'Reinforcement' could correctly classify whether or not healthcare workers received the influenza vaccine. In addition, Williams et al. (2020) found that the 'beliefs about consequences' domain was key in understanding barriers and enablers for receiving a future COVID-19 vaccine in those at high-risk. However, to-date no study has applied the TDF to the understanding of influenza vaccination behaviour amongst the public. Here we address this gap and use the TDF to examine the factors associated with compliance with influenza vaccinations amongst individuals with a chronic respiratory condition. In addition, following the guidance of the UK Medical Research Council on how to develop complex interventions (O'Cathain et al., 2019) we included expert stakeholder engagement, drawing on Affordability, Practicability, Effectiveness/ Cost-Effectiveness, Acceptability, Safety and Equity (APEASE) criteria to refine our suggestions for intervention content.

The present study has four key research questions:

1. What are the factors that shape participants' compliance with influenza vaccination recommendations, and which are the dominant theoretical domains shaping vaccination behaviour?

2. What key themes are present within the most dominant theoretical domains and what do these suggest about the tailoring of future intervention content?

3. What are the relevant intervention functions and associated behaviour change techniques that we can use to provide potential evidence-based and theoretically informed future intervention content?

4. What changes/adaptions do expert stakeholders suggest to ensure intervention content is fit for the future?

\section{Methods}

\section{Design}

This study included qualitative data collected from three sources: open-ended responses from a cross-sectional survey, qualitative data from focus groups, and qualitative data from one-to-one interviews. All data collection was completed before the start of the coronavirus disease (COVID-19) pandemic. The current article comes from a broader exploratory mixed methods study designed to identify the barriers and enablers to seasonal influenza vaccination uptake in adults with chronic respiratory disease. The full details of the methods and results, including further qualitative analysis, are available (Williams et al., 2021).

\section{Sample and procedure}

Ethics approval for this study was received from the University Ethics Committee. A purposive sampling approach was utilised with a convenience sample of adults aged 18-64years, living with at least one self-reported chronic respiratory condition. Interested individuals could participate in qualitative (e.g. focus group or individual interview) or 
Table 1. Summary of participant characteristics.

\begin{tabular}{|c|c|c|c|}
\hline \multicolumn{2}{|c|}{ Independent variable } & $\begin{array}{l}\text { Focus group and } \\
\text { interview participants } \\
(n=59)\end{array}$ & $\begin{array}{l}\text { Open-ended survey } \\
\text { response participants } \\
(n=101)\end{array}$ \\
\hline \multicolumn{2}{|c|}{ Mean age ( \pm Standard Deviation) } & $42.8( \pm 14.8)$ & $40.7( \pm 12.8)$ \\
\hline \multirow[t]{3}{*}{ Gender } & Female & $41(70 \%)$ & $67(60 \%)$ \\
\hline & Male & $18(30 \%)$ & $31(37 \%)$ \\
\hline & Non-binary & - & $3(3 \%)$ \\
\hline \multirow{4}{*}{$\begin{array}{l}\text { Type of chronic } \\
\text { respiratory } \\
\text { condition }\end{array}$} & Asthma & $42(72 \%)$ & $84(83 \%)$ \\
\hline & $\begin{array}{l}\text { Multiple chronic } \\
\quad \text { respiratory conditions }\end{array}$ & $9(15 \%)$ & $5(5 \%)$ \\
\hline & COPD & $6(10 \%)$ & $8(8 \%)$ \\
\hline & Other & $2(3 \%)$ & $4(4 \%)$ \\
\hline \multirow{3}{*}{$\begin{array}{l}\text { Five-year influenza } \\
\text { vaccination uptake }\end{array}$} & Non-vaccinating & $14(24 \%)$ & $41(42 \%)$ \\
\hline & Occasional & $21(36 \%)$ & $47(47 \%)$ \\
\hline & Continuous & $24(41 \%)$ & $13(13 \%)$ \\
\hline
\end{tabular}

quantitative (e.g. cross-sectional survey) data collection but could not participate in both components of the study. This was to avoid introducing potential bias into the study as exposure to the qualitative research questions could influence responses provided in the survey. All participants were recruited from third-sector organisations, respiratory support groups, social media (Facebook and Twitter) posts, advertisements in newsletters and newspapers, public engagement events, and a market research company. We sought to recruit those participants who over the previous five years had always vaccinated, occasionally vaccinated, and those who never vaccinated. We took this approach in order to learn from those people who do regularly comply, those who sometimes comply, and those who do not comply, in order to transfer insights about what does work (enablers) and understand in detail what does not (barriers). The focus group and interview stage of data collection comprised 59 participants, 38 who participated across nine in-person focus groups and 21 in individual interviews. There was a mean of four participants per focus group. Fourteen interviews were conducted via telephone and seven conducted in-person. The mean age of participants was 42.8 (SD \pm 14.8) years and $70 \%$ of participants were females. In addition, 101 participants provided responses to the open-ended survey question. Respondents had a mean age of 40.7 (SD: \pm 12.8 ) years and $60 \%$ were female. Additional demographic details and vaccination behaviours of participants can be found in Table 1 .

\section{Survey comments}

An online cross-sectional survey was used to identify participants' socio-demographic details, attitudes towards vaccinations (not reported in the current paper), and uptake of the influenza vaccine over the previous five years. Participants could provide a qualitative response to an open-ended question ('If there are any reasons why you have not received the flu vaccination that the survey has not addressed, please share them below'). Participants completed the survey online through Qualtrics software.

\section{Focus group and interviews}

Semi-structured focus groups and interviews were conducted using a 13-question interview guide to explore the attitudes of individuals with chronic respiratory conditions towards influenza vaccination. Prompts were also developed for each question 
to further examine characteristics of influenza vaccine hesitancy. The interview guide was pilot tested with a focus group comprised of three female participants with chronic respiratory conditions prior to data collection to ensure question clarity and to determine any changes required to the guide. Participants were sent an email invitation to participate in a focus group and were offered the option of completing an individual interview if they were unable to attend. All focus groups and interviews were facilitated by KD and assisted by AG, two experienced qualitative researchers, and all individual interviews were completed by KD. Audio recordings of focus groups and interviews were transcribed verbatim and anonymised. The mean focus group length was 49:27 minutes and mean interview length was 15:02 minutes.

\section{Data analysis}

A figure providing a summary of data analysis procedures used to identify, develop and prioritise recommendations for improving influenza vaccination uptake is presented in the supplementary files.

1. What are the factors that shape participants' compliance with influenza vaccination recommendations, and which are the dominant theoretical domains shaping vaccination behaviour? To address the first research question, survey responses were exported from Qualtrics software to Microsoft Excel 2016 for analysis. A directed content analysis approach was used (Hsieh \& Shannon, 2005), with responses being mapped onto the TDF. Two coders independently reviewed each response and coded it to the most relevant TDF domain. If a response included multiple influences on the uptake of the vaccine, it was reviewed by the coders and mapped to two TDF domains when necessary. The coders met after coding every 20 responses to review coding, identify and discuss any concerns, and to achieve consensus. If coding consensus could not be achieved for a comment, a third team member resolved the discrepancy. Following each meeting, a master copy of the coding was updated to reflect any changes to coding and discrepancy resolutions. Once all responses were coded, $30 \%$ of responses were independently reviewed by LW and PF. Consensus in coding was achieved throughout. Transcripts from the focus groups and interviews were imported into NVivo 12 software for analysis (QSR International Pty Ltd., 2020) and also subjected to a directed content analysis following a similar process to that outlined above whereby two coders coded each transcript and mapped participant responses to relevant TDF domains. Quotes could be mapped to two domains where coders deemed it appropriate. Coders met consistently after coding every second focus group transcript and after every third interview transcript to discuss and review coding. Coded files were merged in NVivo and coding stripes were compared. Discrepancies in coding were reviewed and discussed to achieve consensus. When consensus could not be achieved, quotes were passed to LW to resolve. Coding resolutions were saved in the NVivo file. Throughout analysis a codebook was developed to note discussions relating to the TDF and coding associated with each domain. Development of the codebook 
was an iterative process and was updated following each coding meeting to reflect discussions and coding resolutions. Following coding, LW and PF reviewed $10 \%$ of coded quotes to ensure accuracy of coding in relation to the appropriate TDF domain.

2. What key themes are present within the most dominant theoretical domains and what do these suggest about the tailoring of future intervention content? To address the second research question, inductive thematic analysis was applied within each TDF domain to determine the overarching themes and subthemes which influenced influenza vaccination behaviours (Atkins et al., 2017). This inductive analysis is a recommended analysis approach suggested when using the TDF by Atkins et al. (2017). Key TDF domains from both the survey comments data and focus group and interview transcripts were identified based on the frequency of content coded under each domain. Major themes and subthemes within domains were ranked based on the number of participants identifying the theme and the number of comments or quotes associated with each theme; this established our sense of which themes were key. Following TDF mapping and inductive thematic analysis of the three streams of qualitative data, data were combined to articulate the commonly identified barriers and enablers from across data these sources.

3. What are the relevant intervention functions and associated behaviour change techniques that we can use to provide potential evidence-based and theoretically informed future intervention content? For the third research question, the key barriers and enablers identified from the analysis relating to the second research question were mapped to the intervention functions level of the BCW. Each factor was compared to the nine intervention functions (e.g. Education, Training, Persuasion) to determine which function(s) would best address the identified barrier or enabler. The BCW intervention functions can be mapped to the BCTT to help identify the 'active ingredients' of interventions targeting behaviour change (Michie et al., 2013, Michie et al., 2014). BCTs from the BCTT were identified to address the relevant $\mathrm{BCW}$ intervention functions and were operationalised to inform recommendations for intervention content to improve influenza vaccination uptake. This work was conducted by AG and audited by PF and LW.

4. What changes/adaptions do expert stakeholders suggest to ensure intervention content is fit for the future? Following the development of initial theory and evidence-informed recommendations for improving influenza vaccination uptake, a meeting was held with six expert stakeholders to review key recommendations. All stakeholders held key positions within public health and government responsible for the implementation of the influenza vaccination programme in Scotland with remits for policy, strategy and marketing. The APEASE criteria were applied to each recommendation to help guide discussions to assess the feasibility and appropriateness of the recommendations in addressing the barriers to influenza vaccination uptake in adults with chronic respiratory conditions (Michie et al., 2014). The APEASE criteria involve assessing each recommendation against its potential acceptability, practicability, effectiveness, affordability, side effects and equity (Michie et al., 2014). These criteria 
were reviewed with the expert stakeholders at the beginning of the meeting and were used to facilitate discussions about each of the presented recommendations. Following discussions, the expert stakeholders could categorise the recommendation as relevant, relevant with some modifications, or impractical for improving influenza vaccination uptake among adults with chronic respiratory conditions.

\section{Results}

\section{1: What are the factors that shape participants' compliance with influenza vaccination recommendations, and which are the dominant theoretical domains shaping vaccination behaviour?}

Nine of the 14 TDF domains were identified in the open-ended survey responses, with the majority of responses falling under 'Beliefs about Consequences' (BACons), followed by the 'Environmental Context and Resources' (ECR) and 'Emotion' domains. Eight comments were coded to two TDF domains as the responses included multiple influences on influenza vaccination uptake. In the focus groups and interviews, participants' quotes were mapped to 12 of the 14 TDF domains, with no responses mapped to the 'Skills' and 'Reinforcement' domains. Barriers and enablers amenable to change were primarily grouped under four domains: 'BACons,' 'ECR', 'Social Influences' and 'Emotion'. As these domains were identified across all data sources they were the focus of subsequent BCW intervention function and BCT mapping.

The full results from behaviour change mapping exercises, operationalised BCT recommendations, and stakeholder meeting feedback can be found in supplementary file 1 . The following sections provide a narrative summary of this work.

\section{2: What key themes are present within the most dominant theoretical} domains and what do these suggest about the tailoring of future intervention content?

\section{BACons}

Table 2 outlines key findings and supporting quotes from TDF mapping and thematic analysis. Within the BACons domain, participants' perception of vaccine side effects was the most commonly identified barrier across the data. Following vaccination, participants described experiencing a range of symptoms, from flu-like symptoms and localised pain at the injection site, to significant asthma exacerbations requiring hospitalisation, all of which they attributed to the vaccine. In addition to perceived personal side effects, anecdotal experiences of friends and family who had perceived poor reactions to the vaccine also influenced their decision not to vaccinate.

In contrast to this barrier, three enablers to influenza vaccination uptake were identified within this domain: previous experience of influenza, a sense of protection from illness, and extra protection against the influenza due to underlying health condition. Those who had experienced influenza described their symptoms, including fatigue, body aches and loss of appetite, and that they chose to vaccinate to prevent experiencing this again. Participants also described vaccination provided a sense of 


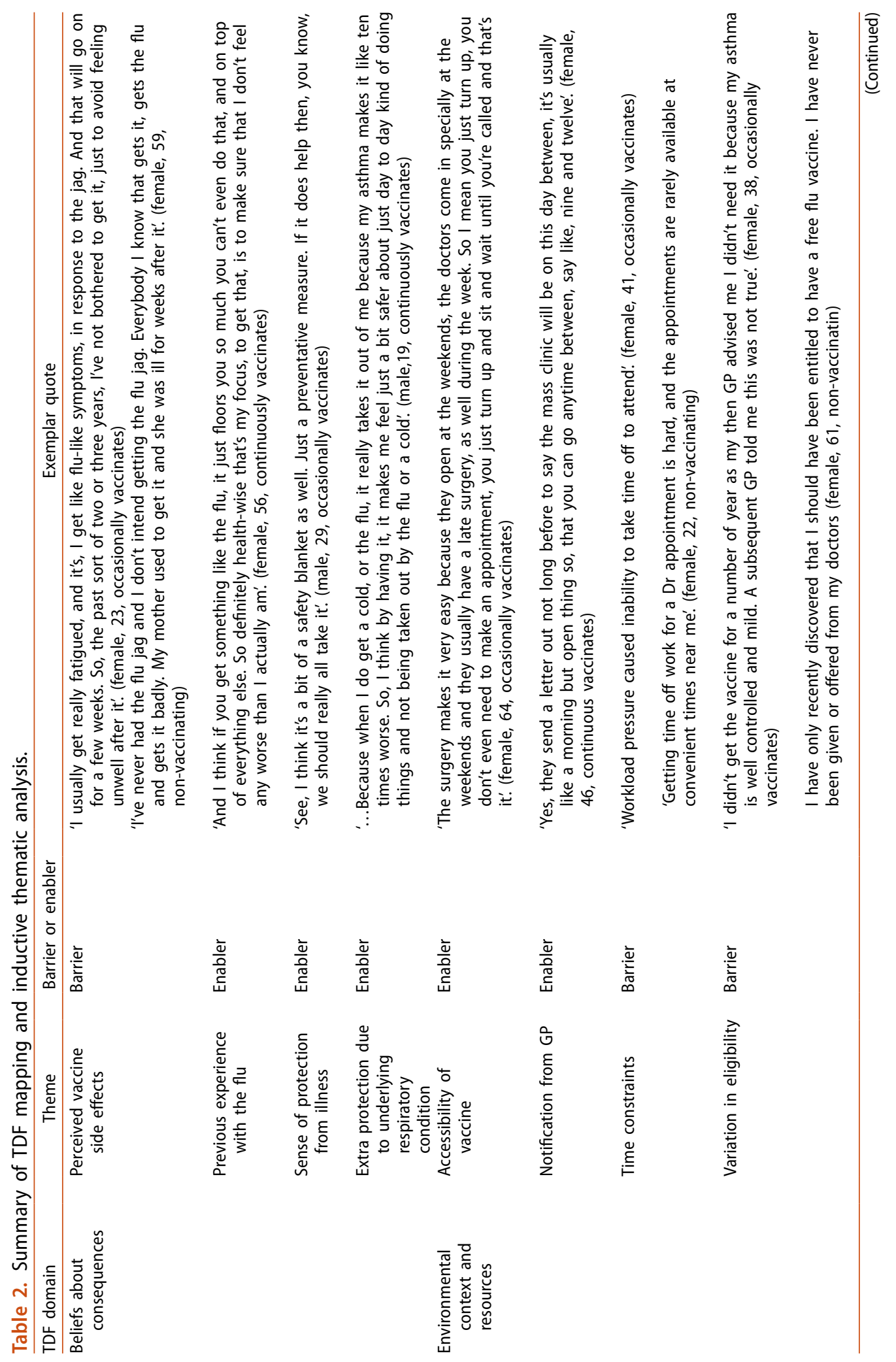




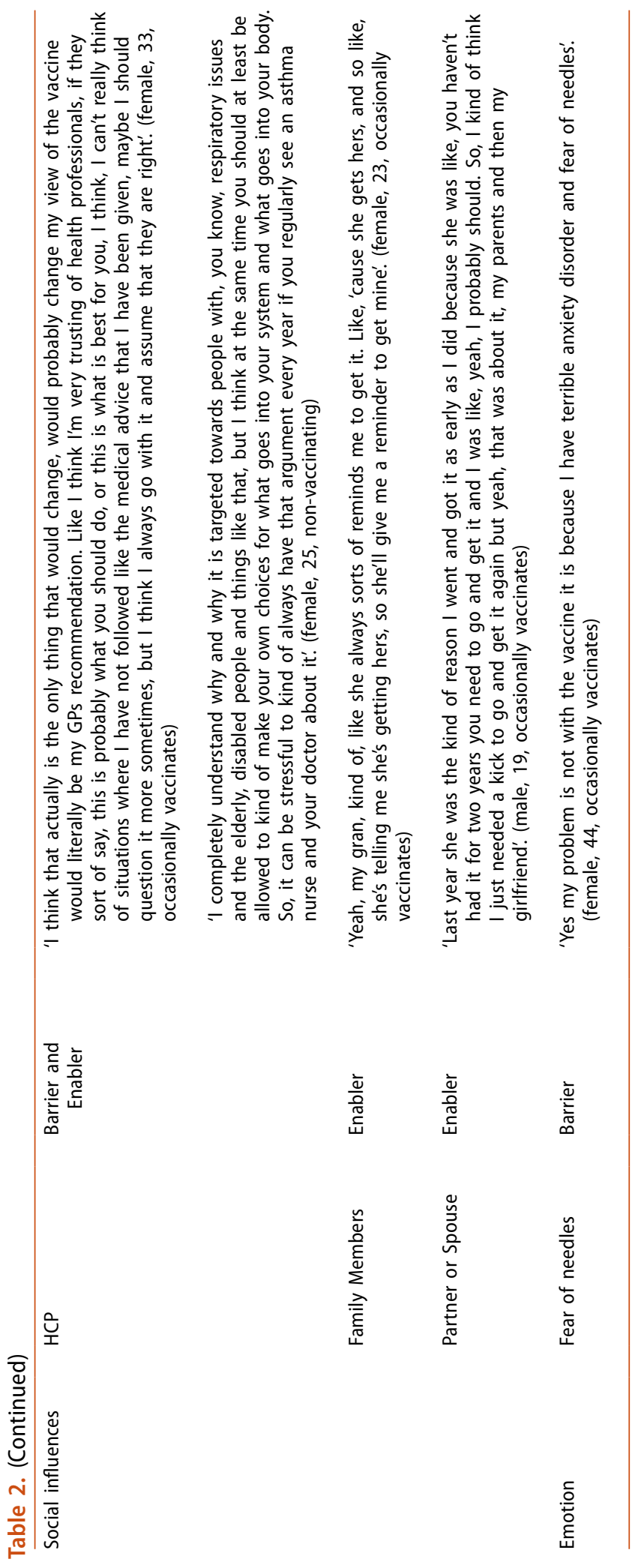


protection or 'safety blanket' against influenza. This sense of protection was also important due to the high-risk status of participants, who felt influenza further exacerbated issues from their chronic respiratory condition.

\section{$E C R$}

Time and geographical constraints, as well as variation in eligibility for the vaccine were two key barriers within this domain. Participants facing busy schedules and limited general practitioner (GP) appointment options made it difficult to prioritise vaccination, especially if they did not work near the practice. Survey participants were more likely to note that they often received confusing advice regarding their eligibility to receive the vaccination for free. Some participants explained they had spent years paying, or being told to pay, for the vaccine as their GP said they were not eligible for the vaccine, only to later learn they had been eligible. This variation in information received from healthcare providers (HCP) limited regular uptake of the vaccine.

Enablers to vaccination within the ECR domain included accessibility of the vaccine, and receiving notifications from their GP. Having GP practices offer out of hours appointments, including evenings and weekends, helped ensure people could vaccinate at a convenient time. Participants also found that receiving a notification (e.g. letter, text message or phone call) from their GP practice served as an important reminder to get vaccinated.

\section{Social influences}

Three key pillars of social support affected vaccination: HCP, family members, and partners or spouses. HCP, including GPs and asthma nurses, could help or hinder vaccination. Those who regularly visited or interacted with these providers felt supported and encouraged to vaccinate, while those who did not feel supported were less likely to vaccinate. Support from family members, particularly parents and grandparents, and partners or spouses was also fundamental in encouraging vaccination.

\section{Emotion}

The main theme identified within the 'Emotion' domain was a fear of needles. Comments described a fear of needles and injections, with some explaining the idea of vaccination was enough to trigger an anxiety or panic attack.

\section{3: What are the relevant intervention functions and associated behaviour change techniques that we can use to provide potential evidence-based and theoretically informed future intervention content?}

Across our BCW intervention function analysis of all the TDF domains outlined above, we identified Education ( $n=8)$, Persuasion $(n=8)$, Enablement $(n=8)$, Environmental Restructuring $(n=4)$, Modelling $(n=2)$, Training $(n=1)$ and Incentivisation $(n=1)$ as important components of interventions aimed at improving influenza vaccination uptake (Table 3 and Supplementary File 1).

Within the BACons domain, interventions should aim to educate patients about the vaccine and its effects through a range of intervention modalities (e.g. mass 


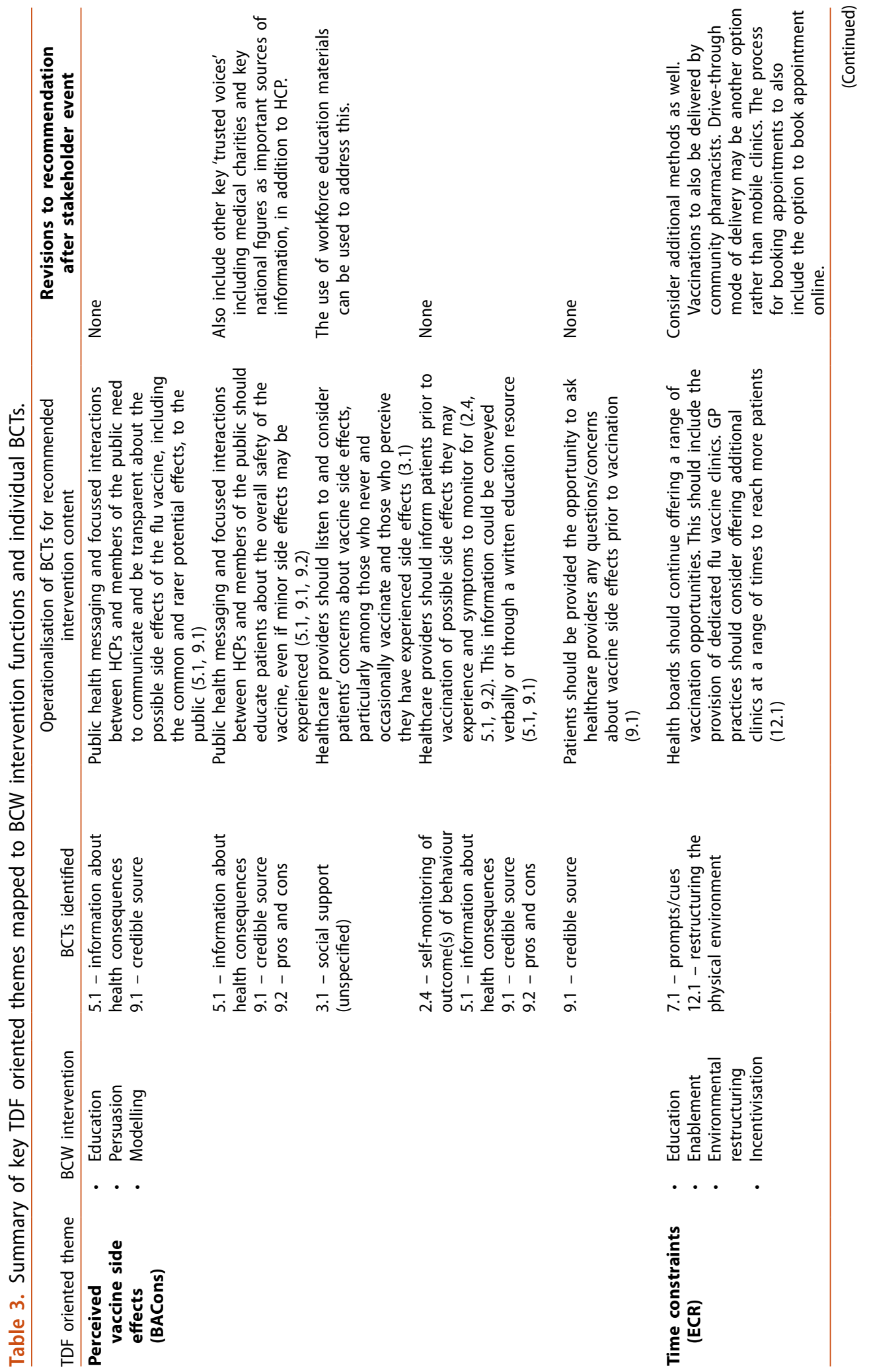




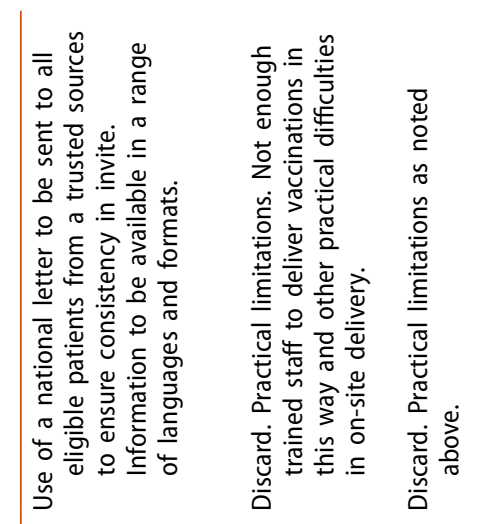

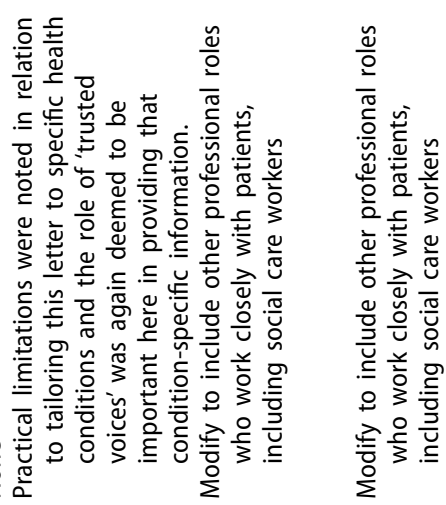

\section{층}

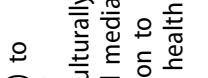

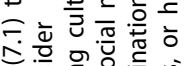

은 원

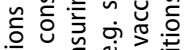

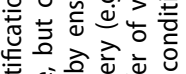

至

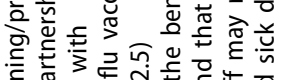

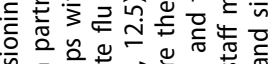

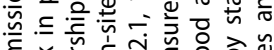

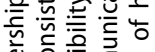

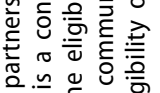

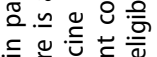

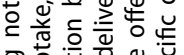

은 늑늄

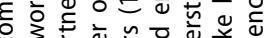

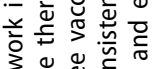

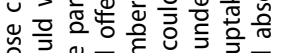

บิ

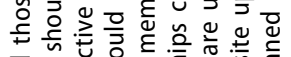

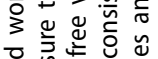

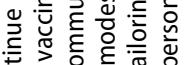

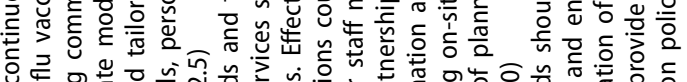

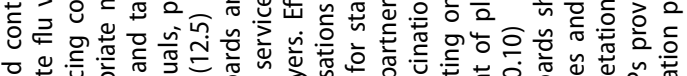

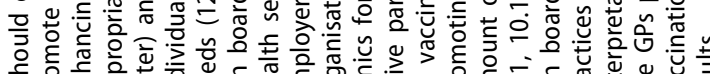

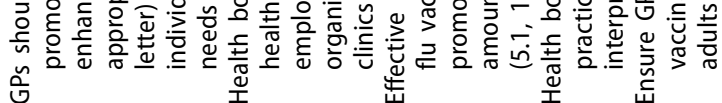

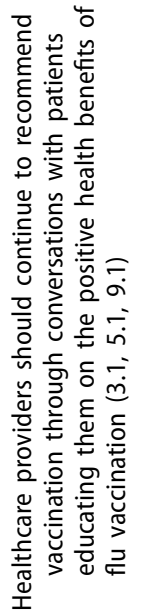

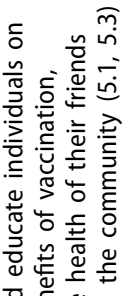

을 离

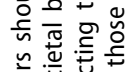

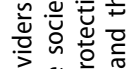

흘 辛 흘

는

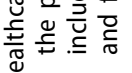

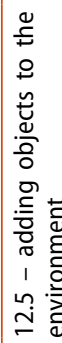

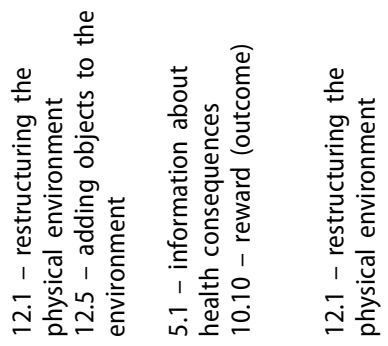

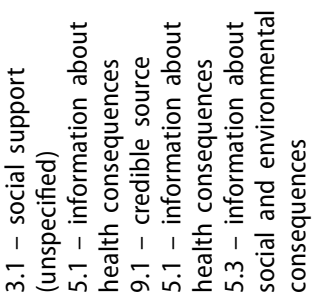
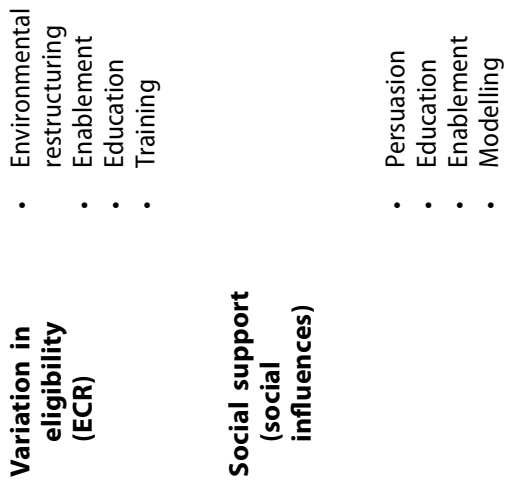


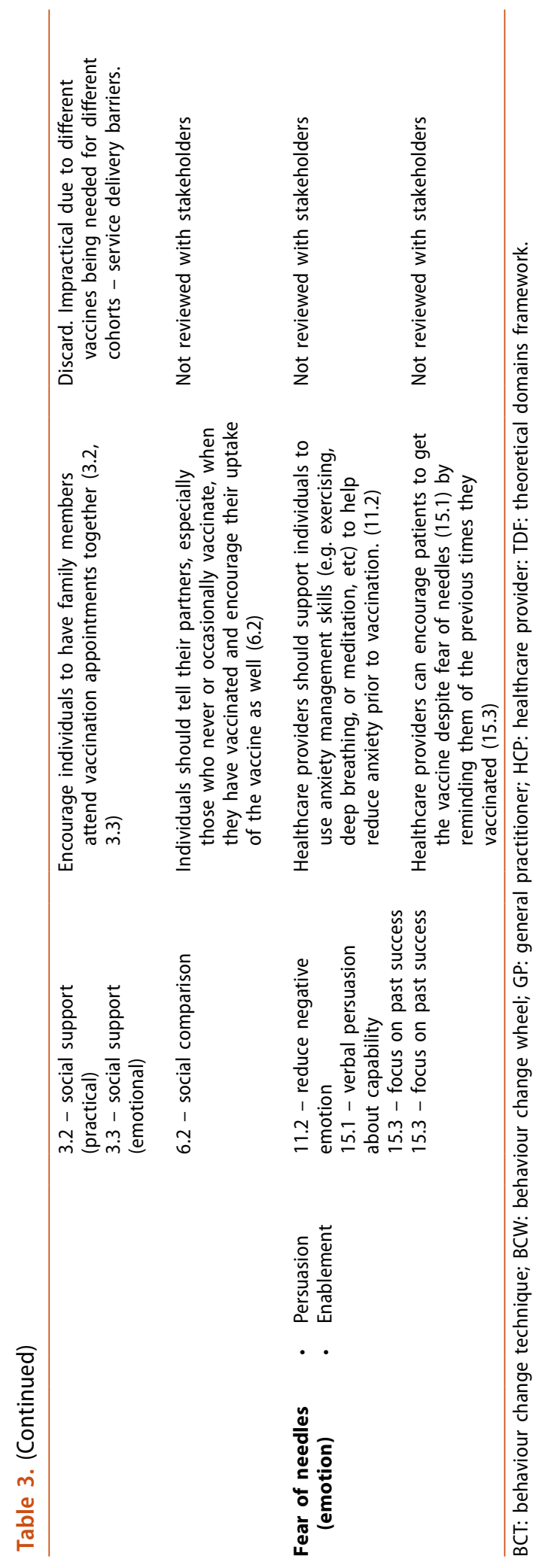


media, social media, interactions with HCPs) should be considered to deliver the intervention functions of Education, Persuasion and Modelling. Overall to directly address BACons, intervention content must redress misinformation, deliver accurate, trustworthy information and provide models of other people's experiences and thinking in order to educate and persuade. These approaches can be delivered within mass and social media and during HCP and patient interactions. Intervention content must provide frank detail of the reality of side effects including explanations of typical immune responses and how these may feel. Consider training for relevant HCPs to deliver brief behaviour change interventions that educate and persuade those showing vaccine hesitancy within every day interactions. These interactions should feature the same decisional balance outlined above and could draw upon principles of motivational interviewing.

To address the environmental barriers to vaccination, interventions should consider Environmental Restructuring, Enablement, Education and Incentivisation to encourage vaccine uptake. Overall, interventions should continue to provide vaccination services in GP practices but there needs to be further emphasis on more community-based vaccination clinics. These could include reimbursing those who paid for their vaccination, incentivising workplaces to provide on-site influenza vaccination clinics, enabling more traditional community-based vaccination clinics, or more novel approaches such as a mobile clinics, in addition to out of hours GP vaccination appointments.

Supportive social influences were a positive influence on vaccination behaviour, which Education, Enablement and Persuasion intervention elements could develop to further encourage uptake. HCP should continue to educate patients on the importance of vaccination and the personal health benefits of vaccinating against influenza, and additional benefits of protecting the health of others by contributing towards herd immunity. Family members and partners could persuade and enable vaccination by attending vaccination clinics together or by using a vaccine 'buddy system'. Finally, fears of needles and injections could be addressed through persuasion and enablement by supporting the use of anxiety-management techniques before and during vaccination appointments.

Applying the BCTT, we operationalised intervention content recommendations to improve vaccine uptake. Recommendations utilised 22 individual BCTs from 11 of the 16 groupings, with the most commonly identified groups including 'Natural Consequences', 'Social Support', 'Antecedents' and 'Comparison of Outcomes'. Table 3 summarises key themes, BCW intervention functions, BCTs applied to our analysis, and how BCTs were operationalised to inform recommendations for intervention content. In Table 3 we present a subset of our recommendations across the key TDF domains.

\section{4: What changes/adaptions do expert stakeholders suggest to ensure intervention content is fit for the future?}

Following the development of recommendations based on the operationalised BCTs, a stakeholder meeting was held with six local expert stakeholders to review 19 key recommendations against the APEASE criteria (for a full list of the recommendations see supplementary file 1). From this meeting, eight recommendations were considered 
relevant to help improve vaccine uptake, six were considered to be relevant with slight modifications, while five were considered impractical to implement. Recommendations that were supported included providing public health messaging of vaccine side effects, encouraging conversations between HCP and patients of potential vaccine side effects prior to vaccination, provision of out of hours vaccination appointments at GP practices and other locations, and routinising the information provided to all high-risk patients regarding the vaccine and their eligibility for free vaccination.

Modifications to recommendations included adding other 'trusted voices' alongside those of HCP as actors within the recommendations, to provide patients with contextualised information relevant to their condition and potential side effects. These 'trusted voices' could include medical charities or national figures, such as the national clinical director, or social care workers. In addition, modifications were suggested to the recommendations relating to vaccine delivery. These included considering other methods such as vaccinations being delivered by community pharmacists, and the potential use of drive-through vaccination centres.

Recommendations deemed impractical by stakeholders due to service-delivery constraints, included providing on-site workplace vaccinations and encouraging a vaccine 'buddy system'. Worksite vaccination services were considered impractical due the limitations of transporting vaccines and vaccination equipment to workplaces and the availability of trained staff to administer the vaccines. The vaccine 'buddy system' recommendation was also considered to be impractical due to the difficulty in having different types of vaccination available to administer at the same time to people from different age cohorts and with different at-risk status.

\section{Discussion}

The present study is the first to apply the BCW and associated BCTT and APEASE tools to the problem of low influenza vaccination uptake among high-risk members of the public. Specifically, we triangulate diverse data sources to identify barriers and enablers to seasonal influenza vaccine uptake in adults with chronic respiratory conditions. By applying these tools we were able to conduct a comprehensive analysis of the factors affecting vaccination behaviour, and develop a range of evidence and theory-informed intervention recommendations to improve vaccine uptake. Working with stakeholders direct responsible for the National seasonal influenza vaccination programme a shorter list of actionable recommendations was agreed. Our findings indicate that influenza vaccination is often a complex decision affected by multifaceted individual, social and environmental influences and future intervention components should address this complexity accordingly.

TDF analysis identified 'Beliefs about Consequences', 'Environmental Context and Resources', 'Social Influences' and 'Emotion' were the most common TDF domains which affected influenza vaccination. Similarly, Williams et al. (2020) also identified 'Beliefs about Consequences' to be the most important domain in relation to barriers and enablers to future uptake of a COVID-19 vaccination. However, our findings contrast from those of Kenny et al. (2020) who examined influenza vaccine uptake in HCP. They found that 'Goals', 'Intentions', 'Social Influences' and 'Reinforcement' 
as significant predictors of influenza vaccine uptake in HCPs working in long-term care. Thus suggesting that the barriers to influenza vaccine uptake experienced by HCPs are different to those faced by patients.

Social Influences is the only factor commonly identified across both studies, which demonstrates the importance of social relationships in vaccine uptake for both HCP and patients. This is consistent with research by Gorman et al. (2012) who analysed factors affecting influenza vaccination in pregnant women. They identified women were three times more likely to vaccinate when they were encouraged to by their healthcare provider, while Kenny et al. (2020) found limited peer support decreased influenza vaccination among HCP. Communication between patients and their HCP provides a noteworthy opportunity to support vaccination. HCP should use their time and interactions with patients, and their position as credible sources of health information, to encourage and support vaccination decisions during every day interactions with patients. In addition, supporting a vaccine 'buddy system' to encourage friends and families to get vaccinated together may also provide an opportunity to capitalise on the role of social support in vaccination behaviour. During the 2019-2020 influenza season, Public Health England encouraged HCP to use a buddy system in an attempt to reach its goal of $90 \%$ vaccination among this group (Ford, 2019). While this recommendation was considered impractical by our stakeholders in Scotland due to the variety of influenza vaccinations available based on age and other risk factors, applying similar systems in communities in other contexts may help address barriers to vaccination and improve uptake.

The findings suggest that perceived vaccine side effects are a predominant barrier to vaccine uptake. Although the influenza vaccine has been shown to be safe and effective, concerns of adverse vaccine events remain (Pebody et al., 2019). Among participants in our study, common side effects including itching and tenderness at the injection site were reported, but infrequent events, including developing influenza, and experiencing eczema and asthma exacerbations, were also attributed to receiving the vaccine. Concerns about vaccine side effects have been well established in influenza vaccine hesitancy research (Schmid et al., 2017; Wiemken et al., 2019), and previous experience with perceived influenza vaccine side effects has also been identified by Ferragut et al. (2020) as a key barrier to vaccination. To help address this concern, HCP should provide open and transparent information about influenza vaccine safety and the prevalence of potential vaccine side effects, from common and less severe symptoms, to the more complex and rare side effects which could be experienced. It is important to provide this information in clear and concise resources, as providing extensive information to those who are vaccine hesitant may have little effect in changing vaccination views or behaviours (Dubé et al., 2020).

Addressing the time and geographical constraints to influenza vaccination is essential in improving uptake of the influenza vaccine. Among our recommendations to offer more out of hour GP appointments during influenza season to improve accessibility of the vaccine, we encourage the use of more traditional community clinics and more novel approaches, such as at workplaces, mobile clinics or drive-through vaccination options. These recommendations to support community vaccination options align with the goals of the Vaccination Transformation Programme in Scotland (VTP; Public Health Scotland, 2020). The VTP was developed by Public Health Scotland 
in 2017 to support the transition of vaccination services, including influenza vaccinations, away from GP practices and towards community-based options through NHS boards. Our suggestions for transitioning influenza vaccination to community-based routes were largely well received by our stakeholders, although our recommendations for workplace vaccination sites were considered to be impractical as there is limited $\mathrm{HCP}$ to conduct this service and difficulty in maintaining vaccine cold chain. The benefits of workplace vaccination have been noted in other countries for improving influenza vaccination rates (Nowalk et al., 2010), and may be useful in some countries for improving accessibility of the vaccine. By providing more accessible vaccination options within communities, it can help address the practical factors to vaccination and allows for more effective use of GP practices and resources.

\section{Strengths and limitations}

The present work adds to the existing literature in a number of ways. The use of multi-modal data collection and subsequent behaviourally informed analysis and expert stakeholder engagement allowed us to develop actionable recommendations. In addition, the use of the TDF mapping approach and the BCW in the current context has a number of advantages. The frameworks most often used to predict vaccination behaviour are the Health Belief Model (Rosenstock, 1974) and the Theory of Planned Behaviour (Ajzen, 1991). Although these models are useful for understanding and predicting behaviour, they focus on a relatively small number of constructs and neglect more contextual and non-conscious processes. It is therefore likely that some aspects of vaccination behaviour are not fully captured by these models. The TDF provides a more comprehensive framework which is useful for vaccination behaviour and allowed us to capture determinants at both the individual level (e.g. Beliefs about Consequences) and the organisational level (e.g. Environmental Context and Resources).

In addition, we provide a comprehensive picture of the barriers and enablers to uptake of the influenza vaccine in those with a chronic respiratory condition by merging qualitative data from multiple sources. Furthermore, we also purposively recruited participants from across the vaccine hesitancy continuum meaning that we were able to learn about the barriers and enablers from both regular and non-vaccinating individuals. By reviewing our recommendations against the APEASE criteria with stakeholders, it helped ensure our recommendations are affordable, practical, effective, applicable and equitable feasible to implement into policy and practice. It was a strength of the project that we could recruit a range of expert stakeholders directly involved within national provision of vaccines and effectively engage them with the project and its findings. Limitations of the stakeholder engagement stage were that all our stakeholders were recruited from a single national organisation. Further honing of the project's final recommendations could have been achieved if we had also recruited from the chain of diverse health care professionals involved in vaccination and attempted to gain wider perspectives from other trusted sources such as the third sector. Other limitations include that our findings are limited to participants living in Scotland with a chronic respiratory condition, and so may not be generalisable to other chronic illnesses or national contexts. Furthermore, data 
were collected before the onset of COVID-19 and it may be that beliefs and behaviours about vaccination have changed radically as a result of this pandemic.

\section{Conclusion}

This study triangulates diverse findings to conduct a comprehensive behavioural diagnosis of compliance with influenza vaccination advice. With the help of stakeholder engagement we have shown that the factors affecting vaccine uptake were multifaceted and multileveled. They could be theorised as relating to the TDF domains of 'Beliefs about Consequences,', 'Environmental Context and Resources', 'Social Influences' and 'Emotion'. The study suggested a suite of complementary multi-level intervention components may be most useful to enhance vaccination compliance involving a range of diverse actors, intervention recipients and settings. Mass and social media interventions, and interactions between recipients and HCP should include clear and concise information about vaccine side-effects and directly address misinformation. Community-based vaccination delivery methods should be enhanced by modifying traditional and adopting novel approaches.

\section{Acknowledgements}

The authors would like to thank Dana O'Flynn for assisting with preliminary TDF coding of survey comments. We would also like to thank the expert stakeholders who participated in our stakeholder meeting for sharing their valuable insights and recommendations.

\section{Disclosure statement}

The authors report no conflict of interest

\section{Funding}

This study was funded by the Chief Scientist Office in Scotland (reference: HIPS 18/37).

\section{ORCID}

Nicola Cogan (D) http://orcid.org/0000-0003-0861-5133

Susan Rasmussen (iD http://orcid.org/0000-0001-6408-0028

Lynn Williams (D) http://orcid.org/0000-0003-2735-9219

\section{Data availability statement}

The qualitative data are not publicly available due to privacy or ethical restrictions.

\section{References}

Atkins, L., Francis, J., Islam, R., O'Connor, D., Patey, A., Ivers, N., Foy, R., Duncan, E. M., Colquhoun, H., Grimshaw, J. M., Lawton, R., \& Michie, S. (2017). A guide to using the theoretical domains framework of behaviour change to investigate implementation problems. Implementation Science: IS, 12(1), 77. https://doi.org/10.1186/s13012-017-0605-9

Ajzen, I. (1991). The theory of planned behavior. Organizational Behavior and Human Decision Processes, 50(2), 179-211. 
Borthwick, C., O'Connor, R., \& Kennedy, L. (2021). Psychological predictors of seasonal influenza vaccination uptake among adults with a high-risk physical health condition: A systematic review. Psychology \& Health, 36(2), 214-222. https://doi.org/10.1080/08870446.2020.1772971

Brewer, N. T., Chapman, G. B., Rothman, A. J., Leask, J., \& Kempe, A. (2017). Increasing vaccination: Putting psychological science into action. Psychological Science in the Public Interest, 18(3), 149-207. https://doi.org/10.1177/1529100618760521

Cane, J., O'Connor, D., \& Michie, S. (2012). Validation of the theoretical domains framework for use in behaviour change and implementation research. Implementation Science, $7(1), 37$. https://doi.org/10.1186/1748-5908-7-37

Craig, P., Dieppe, P., Macintyre, S., Michie, S., Nazareth, I., \& Petticrew, M. (2008). Developing and evaluating complex interventions: The new Medical Research Council guidance. BMJ (Clinical Research Ed.), 337, a1655.

Dubé, E., Gagnon, D., \& Vivion, M. (2020). Optimizing communication material to address vaccine hesitancy. Canada Communicable Disease Report=Releve Des Maladies Transmissibles au Canada, 46(2-3), 48-52. https://doi.org/10.14745/ccdr.v46i23a05

Ferragut, M. J., Barry, D., \& Cummins, M. (2020). Understanding why healthcare workers refuse the flu vaccine. Journal of Infection Prevention, 21(3), 115-118. https://doi.org/10.1177/ 1757177420908000

Ford, M. (2019). Government sets $90 \%$ flu vaccine target for health staff this winter. Nursing Times. https://www.nursingtimes.net/news/public-health/embargoed-new-flu-vaccin e-campaign-sets-90-target-for-health-professionals-04-10-2019/

Gorman, J. R., Brewer, N. T., Wang, J. B., \& Chambers, C. D. (2012). Theory-based predictors of influenza vaccination among pregnant women. Vaccine, 31(1), 213-218. https://doi. org/10.1016/j.vaccine.2012.10.064

Health Protection Scotland. (2020). Seasonal influenza vaccine uptake data visualisation. Retrieved August 14, 2020, from https://www.hps.scot.nhs.uk/a-to-z-of-topics/influenza/

Hsieh, H.-F., \& Shannon, S. E. (2005). Three approaches to qualitative content analysis. Qualitative Health Research, 15(9), 1277-1288. https://doi.org/10.1177/1049732305276687

Jorgensen, P., Mereckiene, J., Cotter, S., Johansen, K., Tsolova, S., \& Brown, C. (2018). How close are countries of the WHO European Region to achieving the goal of vaccinating $75 \%$ of key risk groups against influenza? Results from national surveys on seasonal influenza vaccination programmes, 2008/2009 to 2014/2015. Vaccine, 36(4), 442-452. https://doi.org/10.1016/j. vaccine.2017.12.019

Kenny, E., McNamara, Á., Noone, C., \& Byrne, M. (2020). Barriers to seasonal influenza vaccine uptake among health care workers in long-term care facilities: A cross-sectional analysis. British Journal of Health Psychology, 25(3), 519-539. https://doi.org/10.1111/bjhp.12419

Lawton, R., Heyhoe, J., Louch, G., Ingleson, E., Glidewell, L., Willis, T. A., McEachan, R. R. C., \& Foy, R. (2015). Using the Theoretical Domains Framework (TDF) to understand adherence to multiple evidence-based indicators in primary care: A qualitative study. Implementation Science, 11(1), 113. https://doi.org/10.1186/s13012-016-0479-2

MacDonald, N. E., \& The SAGE Working Group on Vaccine Hesitancy. (2015). Vaccine hesitancy: Definition, scope and determinants. Vaccine, 33(34), 4161-4164. https://doi.org/10.1016/j. vaccine.2015.04.036

Michie, S., Atkins, L., \& West, R. (2014). The behaviour change wheel: A guide to designing interventions (1st ed.). Silverback Publishing.

Michie, S., Richardson, M., Johnston, M., Abraham, C., Francis, J., Hardeman, W., Eccles, M. P., Cane, J., \& Wood, C. E. (2013). The behavior change technique taxonomy (v1) of 93 hierarchically clustered techniques: building an international consensus for the reporting of behavior change interventions. Annals of Behavioral Medicine: A Publication of the Society of Behavioral Medicine, 46(1), 81-95. https://doi.org/10.1007/s12160-013-9486-6

Nowalk, M. P., Lin, C. J., Toback, S. L., Rousculp, M. D., Eby, C., Raymund, M., \& Zimmerman, R. K. (2010). Improving influenza vaccination rates in the workplace: A randomized trial. American Journal of Preventive Medicine, 38(3), 237-246. https://doi.org/10.1016/j.amepre.2009.11.011 
O'Cathain, A., Croot, L., Duncan, E., Rousseau, N., Sworn, K., Turner, K., Yardley, L., \& Hoddinott, P. (2019). Guidance on how to develop complex interventions to improve health and healthcare. BMJ Open, 9(8), e029954. https://doi.org/10.1136/bmjopen-2019-029954

Pebody, R., Djennad, A., Ellis, J., Andrews, N., Marques, D. F. P., Cottrell, S., Reynolds, A. J., Gunson, R., Galiano, M., Hoschler, K., Lackenby, A., Robertson, C., O'Doherty, M., Sinnathamby, M., Panagiotopoulos, N., Yonova, I., Webb, R., Moore, C., Donati, M., ... Zambon, M. (2019). End of season influenza vaccine effectiveness in adults and children in the United Kingdom in 2017/18. Eurosurveillance, 22(44): 17-00306. https://doi.org/10.2807/1560-7917.ES.2019.24.31. 1800488

Public Health Scotland. (2020). Immunisation - Vaccination transformation programme. Retrieved August 14, 2020 from http://www.healthscotland.scot/health-topics/immunisation/ vaccination-transformation-programme

QSR International Pty Ltd. (2020). NVivo (released in March 2020). https://www.qsrinternational. com/nvivo-qualitative-data-analysis-software/home

Rosenstock, I. M. (1974). The health belief model and preventive health behavior. Health Education Monographs, 2, 328-335.

Schmid, P., Rauber, D., Betsch, C., Lidolt, G., \& Denker, M. L. (2017). Barriers of influenza vaccination intention and behavior - A systematic review of influenza vaccine hesitancy, 2005 2016. PloS One, 12(1), e0170550. https://doi.org/10.1371/journal.pone.0170550

Wiemken, T., Ramirez, J., \& Carrico, R. (2019). Reasons for vaccine declination in healthy individuals attending an international vaccine and travel clinic. Journal of Refugee \& Global Health, 2(1), 2. https://doi.org/10.18297/rgh/vol2/iss1/2

Williams, L., Gallant, A. G., Rasmussen, S., Brown Nicholls, L. A., Cogan, N., Deakin, K., Young, D., \& Flowers, P. (2020). Towards intervention development to increase the uptake of COVID-19 vaccination among those at high risk: Outlining evidence-based and theoretically informed future intervention content. British Journal of Health Psychology, 25(4), 1039-1054. https://doi. org/10.1111/bjhp.12468

Williams, L., Deakin, K., Gallant, A., Rasmussen, S., Young, D., \& Cogan, N. (2021). A mixed methods study of seasonal influenza vaccine hesitancy in adults with chronic respiratory conditions. Influenza and Other Respiratory Viruses. https://doi.org/10.1111/irv.12856 